\title{
A REVIEW OF SYBIL ATTACK IN MANET
}

\author{
Harsatnam Singh Atwal ${ }^{1}$, Narinder Kumar Rana ${ }^{2}$ \\ Department of Computer Science and engg, RIEIT.harsatnam90@yahoo.com, \\ Department of Computer Science and engg, RIEIT.narinderkrana@gmail.com.
}

\begin{abstract}
SYBIL attack is a severe security trouble to be solved for successful delivery of packets in mobile adhoc network. In this issue, a mischievous hub that uses steering convention to advance itself as having the littlest way to the hub whose bundles it wants to get. In flood based convention, if the evil hub reaction achieves the asking for hub past to the answer from the genuine hub, a fake course is made. This paper deals with the presentation of SYBIL attack in MANET. Various previous techniques have been discussed in these papers that were used to prevent SYBIL attack. MANETs are vulnerable to a diversity of attacks, so attacks has to be mitigated in initial setup.
\end{abstract}

KEYWORDS: Routing, SYBIL Attack And Security, AODV.

\section{INTRODUCTION}

Remote system is the arrangement of versatile PC hubs or stations that are not really wired. The primary advantage of this is speaking with relief of the world however life forms adaptable. The primary complicatedness is their restricted transmission capacity, memory, preparing capacities and open hub [1]. Two indispensable assembly models that are foreordained spine remote configuration and Wireless Mobile Ad hoc Network (MANET). An untrained system is a situated of hubs that don't depend on a predefined framework to keep the system linked. So the completion of Ad-hoc systems is needy on the trust and widespread support between centers. Hubs assist one another in giving over data about the topology of the framework and partition the good faith of dealing with the system. Hence forth in adding to going in family associate to as has, every portable hub does the extent of directing and handing-off post for another flexible hubs [1]. Most fundamental systems management operations include direction finding and system management [2]. Steering convention can be differentiated into downward to earth, open and cross convention that relies on upon the directing topology. Useful meeting is frequently table obsession. Samples of this type slit in DSDV, WRP. Rushed or source started on interest convention, in division they don't one time in a while reconsider the direction finding in place. It is proliferated to the hubs just when compulsory. A container of this sort incorporate DSR; AODV and ABR. consolidate conventions make utilization of both receptive and functional methodologies. Examples of this sort incorporate TORA, ZRP. Comfort is a real stress in all types of communication systems, yet specially agreed system face the greatest challenge because of their intrinsic nature. Subsequently, there exist swings of assaults that can be performed on an Ad hoc system. [1][4].But this protocol does not work well so this paper contains the beginning part to swarm optimization algorithms.

This paper shows the idea of SYBIL attack in MANET in addition to SYBIL prevention methods.

\subsection{SYBIL ATTACK}

The SYBIL attack is individual type of association layer attack in Mobile specially chosen Network. In this attack, a false hub presents itself that has direct path to reach target [3]. So it collects the whole packet from source and drop it. The Sybil attack is off two types:

a) Single SYBIL attack: In Single SYBIL attack, only one node acts as a false node that collects the entire packet from source and drops the packet [4]. The solitary SYBIL attack is shown in Fig.1.1. The basis node $S$ wants to talk with purpose D. Initially, it sends RREQ to the national node. If it has appropriate route to reach destination then it sends the packet throughout the path. If it does not clasp a path then it ahead's the RREQ to the neighbor's node until attain destination. The enlargement $\mathrm{F}$ perform as a forged node that send RREP with maximum sequence number before any other node react, even if any intermediary node send RREP to the source. The basis node $\mathrm{S}$ discards the reply and it assumes that the $\mathrm{F}$ node has direct path to reach Destination and it sends the packet from end to end on that path. So, the node F collects all the packets coming from founded node which creates SYBIL problem.

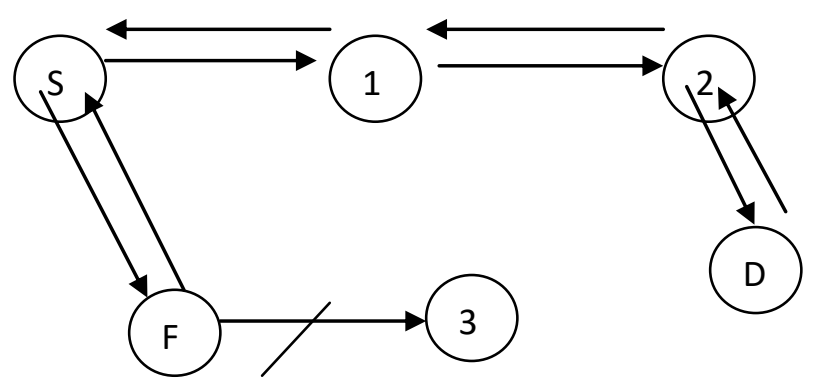

Figure 1.1 Single SYBIL Attack 
b) Co-operative SYBIL attacks: In Co-operative Sybil attack, extra than one node combined equally and act as Fake nodes is called as sympathetic Sybil attack [4]. This determination put down the system form. The Co-operative Sybil attack is shown in Fig.1.2.

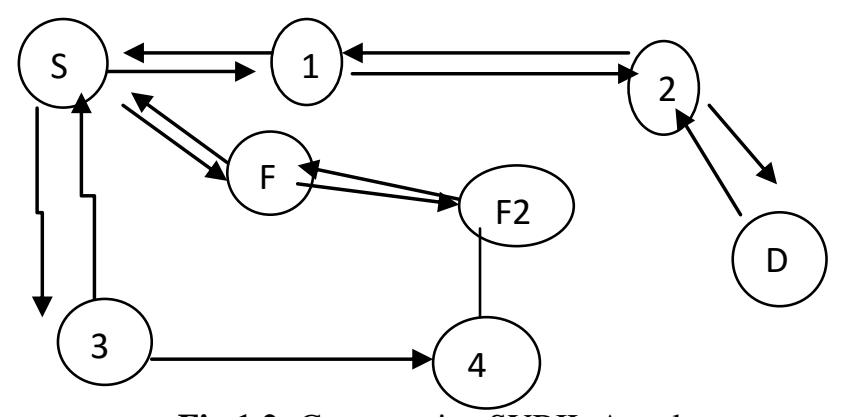

Fig 1.2: Co-operative SYBIL Attack

\section{LITERATURE SURVEY}

Crosbie ET. Al (1995) presented a probable result to the interruption recognition problem in computer security. It uses a contract of work in the field of Artificial Life and computer security. It shows how an interference detection system can be developed using self-governing agents, and how this agent can be built using hereditary Programming. It also shows how Automatically Defined Functions that can be used to develop hereditary programs that contain several data types and however hold type-safety. [6]

Chun Hu et.al (2006) presented the wormhole assault, an unforgiving assault in specially appointed systems that is chiefly hard to protect beside. The wormhole attack is maybe regardless of the possibility that the interloper has no cooperation with any of the hosts and regardless of the opportunity that each message gives legitimacy and deliberateness. In the wormhole attack, an invader accounts packet at one position in the network, tunnel them to a unlike position, and retransmits them there into the support. The wormhole assault can shape a perceptive risk in remote systems, for the most part against numerous specially appointed system steering conventions and area based remote security frameworks. They illustrate a well-known stuff, called package rope, for recognize and, hence self suspicious against wormhole assaults, they in adding up contend topology based wormhole revelation, and display that it is not viable for this way to deal with declaration some wormhole topologies. [7]

Marianne Azeret.al (2009) discussed troubled of mainly harsh security attacks that have an effect on the expressly appointed systems directing convention, it is known as the wormhole physical attack. Wormhole assault is a two stage procedure dispatch by one or a few awful hubs. In the first stage, these terrible nodes, entitled as wormhole nodes, try to pull in legitimate hubs to toss information to different hubs through them. In the second stage, wormhole hubs could develop the information in mixture of ways. It will get in the wormhole assault modes and classes, and point to its effect and threat on impromptu systems. We likewise examine the wormhole assault modes from an assailant's perspective and propose new enhancement to this type of attacks. We finally reduce and end this document [8].

Tandanand Saurabh (2011) An Ad hoc network is the complex with no fixed communications. There is no middle manager so any node can come and move in and exterior of the network in a dynamic way. This makes it more active and complex which makes it more vulnerable to attacks. A few effects of malicious nodes are rejection of service, Routing table flood, Pretense, consumption of Energy, Info revelation etc. A SYBIL attack node attracts all bundles by mistakenly guaranteeing a new course to the destination hub and assimilates them without forwards them to destination. In this paper apparatus based on PDRR is proposed to detect the SYBIL attack in MANET with AODV procedure. A foreword of SYBIL in MANET with QUALNET 5.0 is done, after apply the detection technique result reflect the performance degradation. This paper is future for audience having previous knowledge about network routing protocols and its related quantitative presentation metrics. [9]

Ira NathandRituparnaChaki (2012) SYBIL attack is single variety of direction finding exasperating assaults and can convey awesome harm to all groups of a MANET. Security buildups a major stand up to for these system because of their facial development of open medium, effectively changing topologies, and property without base. As an outcome, a trained calculation to notice SYBIL assault is vital. This paper proposes and assesses procedure for recognizing SYBIL assaults and create reliable and protected bury bunch steering in remote impromptu network.[10]

Amolet.al (2012) Mobile impromptu system is a selfarranging framework that is framed naturally by means of remote connections by a gathering of portable hubs with the assistance of a settled interchanges or focal association. Because of enthusiastic foundation less nature and absence of concentrated check focuses, the impromptu systems are powerless against assaults. The system execution and steadiness is break by assaults on impromptu system directing conventions. AODV is a major on interest rushed directing convention for portable spontaneous systems. There is no any security condition against a "SYBIL" and "Wormhole" assaults in possible AODV convention. SYBIL hubs are those offensive hubs that comply with forward packet to reason. In any case, they don't onward parcel intentionally to the destination hub. The SYBIL hubs corrupt the sign of system in the end by participates in the system successfully. The motivation behind choice making body system is to make out the SYBIL hubs in a MANET. These routine first identify a SYBIL mugging in the framework and afterward supply another line to this hub. In this, the attendance of unique AODV and adjusted AODV in the going to of incessant SYBIL hubs is finding out on the premise of throughput and bundle free proportion. In a wormhole assault, intruder passage the information from one end of the system to the next, most essential secluded organization hubs to confidence they are neighbors' and manufacture them impart through the wormhole link.[11] 
Trupti et.al (2012) MANET comprises of mobile hosts furnish with remote message gadgets. A Mobile Ad hoc Network is a self-sorting out, affecting less, multi-jump complex the telecast of a versatile host is traditional by all hosts inside its transmit run because of the pass on nature of remote correspondence and unidirectional reception apparatus. On the off chance that two remote hosts are out of their system runs in the specially appointed systems, other cell phone hosts located between them can ahead their message, which professionally fabricate coupled systems among the portable has in the sent range. One principle challenge in outline of these systems is their helpless nature to security assault. These assaults can be sending by inside attacker or odder aggressor. A tad bit, the hub from system can be assailant. This happen due to portability of hubs and shifting system topology. There are typical sort of assaults accessible in composing and can be executing on MANET. One of these assaults is Gray Hole assault that has dodgy result on Mobile ad-hoc Network. This paper talked about the final result of Gray Hole assault on Dynamic Source Routing convention in Mobile ad-hoc Network. [12]

\section{PREVIOUS TECHNIQES USED}

\begin{tabular}{|c|c|c|}
\hline AUTHOR NAME & TECHNIQUE & DESCRIPTION \\
\hline Marco [13] & $\begin{array}{l}\text { ABC(Artificial } \\
\text { Colony) }\end{array}$ & $\begin{array}{l}\text { The ABC algorithm is a grouping based algorithm based on } \\
\text { the foraging actions of bumble bee provinces. It is a simple, } \\
\text { strong and populace based stochastic reorganization } \\
\text { calculation. The manifestation of the ABC calculation is } \\
\text { contrasted and the other surely understood current heuristic } \\
\text { calculations, for example, Differential development, Particle } \\
\text { Swarm Optimization on controlled and free issues. }\end{array}$ \\
\hline $\begin{array}{l}\text { Sowmya K.S, RakeshT. and } \\
\text { Deepthi P Hudedagaddi [14] }\end{array}$ & $\begin{array}{ll}\text { ACO(Ant } & \text { Colony } \\
\text { Optimization) } & \end{array}$ & $\begin{array}{l}\text { The essential guideline of a ground dwelling insect directing } \\
\text { calculation is that ants drop a Harmon on the belief, known as } \\
\text { the pheromone, while they travel searching for nourishment. } \\
\text { Ants can likewise notice pheromone and tend to take after } \\
\text { with higher prospect those ways described by solid } \\
\text { pheromone concentration. }\end{array}$ \\
\hline PreetiGulia [15] & $\begin{array}{l}\text { BFO(Bacterial } \\
\text { Optimization) }\end{array}$ & $\begin{array}{l}\text { The technique BFOA (bacterial foraging optimization } \\
\text { algorithm) is new comer to the genetic techniques. The } \\
\text { procedure, in which a microbes moves by making little strides } \\
\text { while cutting for supplements, is called chemo taxis and key } \\
\text { thought of BFOA is imitating chemo strategy brotherhood of } \\
\text { commonsense microscopic organisms in the subject hunt } \\
\text { space, individual bacterium swap over a few words to other } \\
\text { by transmitting signals. It is a worldwide optimization } \\
\text { algorithm for a variety of optimization problems. This } \\
\text { methods likewise animated by the social searching conduct } \\
\text { like burrowing little creature settlement and molecule swarm } \\
\text { improvement. It draws in the specialists because of its skill in } \\
\text { tackling true enhancement issues and gives preferable results } \\
\text { over regular techniques for issues unraveling. }\end{array}$ \\
\hline RichaKalucha [16] & $\begin{array}{l}\text { PSO (Particle } \\
\text { optimization) }\end{array}$ & $\begin{array}{l}\text { Particle swarm optimization (PSO) is a stylish } \\
\text { multidimensional development system [9]. ease of fulfillment } \\
\text { high caliber of arrangements, computational productivity and } \\
\text { rate of meeting are qualities of PSO. PSO has been a famous } \\
\text { technique used to tackle advancement issues in WSNs } \\
\text { because of its effortlessness, high caliber of arrangement, } \\
\text { quick meeting and immaterial computational weight. }\end{array}$ \\
\hline
\end{tabular}

\section{CONCLUSION}

MANETs is quiet unsafe as well as susceptible to numerous attacks so it is necessitate a dependable, proficient as well as a protected protocol which can be able to quickly organized and also utilize dynamic routing technique. Sybil attacks in wireless can be forbidden using different protocols and optimization algorithms so that data can be firmly transferred from source to goal. Sybil attack is a huge risk to the security of mobile ad-hoc networks. A variety of technique has been discussed and each has its own significance. 


\section{REFERENCES}

[1]. Cai, Jiwen, et al. "An adaptive approach to detecting black and gray hole attacks in ad hoc network." Advanced Information Networking and Applications (AINA), 2010 24th IEEE International Conference on. IEEE, 2010.

[2]. Alem, YibeltalFantahun, and Zhao Cheng Xuan. "Preventing SYBIL attack in mobile ad-hoc networks using Anomaly Detection." Future Computer and Communication (ICFCC), 2010 2nd International Conference on. Vol. 3. IEEE, 2010.

[3]. Bhosle, Amol A., Tushar P. Thosar, and SnehalMehatre. "Black-hole and wormhole attack in routing protocol AODV in MANET." International Journal of Computer Science, Engineering and Applications (IJCSEA) Vol 2 (2012).

[4]. Vennila, G., D. Arivazhagan and N. Manickasankari. "Prevention of Co-operative SYBIL attack in Manet on DSR protocol using Cryptographic Algorithm." G. Vennila et al./International Journal of Engineering and Technology (IJET), ISSN (2014): 0975-4024.

[5]. Rao, DB Jagannadha, KarnamSreenu, and ParsiKalpana. "A Study on Dynamic Source Routing Protocol for Wireless Ad Hoc Networks." International Journal of Advanced Research in Computer and Communication Engineering1.8 (2012): 2319-5940.

[6]. Crosbie, Mark, and Gene Spafford. "Applying genetic programming to intrusion detection." Working Notes for the AAAI Symposium on Genetic Programming. MIT, Cambridge, MA, USA: AAAI, 1995.

[7]. Hu, Yih-Chun, Adrian Perrig, and David B. Johnson. "Wormhole attacks in wireless networks." Selected Areas in Communications, IEEE Journal on 24.2 (2006): 370-380.

[8]. MarianneAzer ,Sherif El-Kassas and Magdy ElSoudani Attacks, Towards Introducing Complex Wormhole. "A Full Image of the Wormhole Attacks." (2009).

[9]. Saurabh, ShekharTandanandPraneet. "A PDRR based detection technique for blackhole attack in MANET.",2011.

[10]. Nath, Ira, and DrRituparnaChaki. "BHAPSC: A New SYBIL Attack Prevention System in Clustered MANET." International Journal of Advanced Research in Computer Science and Software Engineering 2.8 (2012): 113-121.

[11]. Bhosle, Amol, TusharThosar and SnehalMehatre. "Black-hole and wormhole attack in routing protocol AODV in MANET." International Journal of Computer Science, Engineering and Applications (IJCSEA) Vol 2 (2012).

[12]. PATEL, Tripti, RANI C. SHYAMALA, and H. PATEL. "Performance evaluation of DSR protocol under DoS attack." International Journal of Electronics and Computer Science Engineering 1.2 (2012): 9-14.
[13]. De Oca, Marco A. Montes,"A comparison of particle swarm optimization algorithms based on run-length distributions." Ant Colony Optimization and Swarm Intelligence. Springer Berlin Heidelberg, 2006. 1-12.

[14]. Sowmya, K. S., T. Rakesh, and P. HudedagaddiDeepthi. "Detection and Prevention of Blackhole Attack in MANET Using ACO." International Journal of Computer Science and Network Security 12.5 (2012): 21-24.

[15]. Gulia, Preeti, and SumitaSihag. "Review and Analysis of the Security Issues in MANET." International Journal of Computer Applications 75.8 (2013): 23-26.

[16]. Kalucha, Richa, and Deepak Goyal. "A Review on Artificial Bee Colony in MANET." (2014).

\section{BIOGRAPHIES}

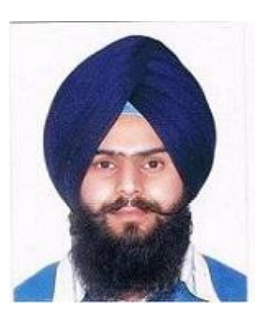

Harsatnam singh Atwal received his bachelor's in technology from Punjab technical university in the department of information technology and pursuing M.tech from Punjab technical university in the department of computer science. His principal research interests include security issues in Mobile ad hoc networks, intrusion detection \& prevention, and routing in Sensor Networks.

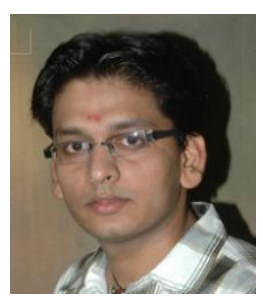

Narinder kumar rana received his bachelor's and master's in technology from Punjab Technical university in the department of computer science with distinction and currently pursuing P.H.D.His principal research interests include digital image processing and mobile ad hoc networks. He is an assistant professor in the Department of Computer Science at RIEIT affiliated to Punjab technical university. 